\title{
Mapping the Milky Way: A Radio Astronomy-Directed Investigation for Lecture-Based Astro 101 Courses
}

Kathryn Williamson ${ }^{1 *}$, Dan Reichart ${ }^{2}$, Colin Wallace ${ }^{2}$, Edward E. Prather ${ }^{3}$, and Seth Hornstein ${ }^{4}$

\begin{abstract}
The Green Bank 20-meter radio telescope integrated into the Skynet Robotic Telescope Network offers a unique opportunity to engage learners in investigations that are not possible with optical telescopes. Radio investigation of the Milky Way using the neutral hydrogen $(\mathrm{HI})$ 1420.41 MHz emission line have a long history with the educators at Green Bank Observatory and through the Skynet team. The project discussed considers how best to adapt these investigations into a large-enrollment, general education, introductory college astronomy course ("Astro 101"). Astro 101 courses serve over 250,000 students nationwide each year. To conduct a class-wide investigation of the Milky Way, we first have each student collect a 60-second radio spectral scan of the $\mathrm{HI}$ emission line for a single portion of the galactic disk. Once this class data over a wide range of galactic longitudes is combined, the students can use simple geometry and Doppler information to determine that we live in a spiral galaxy and that we orbit the galactic center clockwise (if looking down on the Galactic North Pole). Together with archived data of the Large and Small Magellanic Clouds, a galactic rotation curve and an enclosed mass curve can be created to illustrate the "missing mass" problem as evidence for existence of dark matter. Results from a formative assessment illustrate that this project helps students connect concepts learned in class and gain confidence in their abilities to do scientific research. Activity lecture slides and data spreadsheets are freely available.
\end{abstract}

\section{Keywords}

radio astronomy; Skynet; Milky Way; Astro 101

${ }^{1}$ West Virginia University

${ }^{2}$ University of North Carolina

${ }^{3}$ University of Arizona, Center for Astronomy Education

${ }^{4}$ University of Colorado, Boulder

*Corresponding author: kewilliamson@mail.wvu.edu

\section{Introduction}

The introductory college astronomy course, "Astro 101 ," is an important avenue for engaging a wide-range of students in science, especially non-science majors and future teachers (Partridge and Greenstein, 2003; Rudolph et al., 2010), physical laws and subjects such as gravity and spectra, and the roles and degrees of uncertainty) as well as skills, values, and attitudes (ex: excitement of actually doing science, analyzing evidence, critical thinking and quantitative reasoning, and increased feelings of inspiration and confidence) (Partridge and Greenstein, 2003).

Meeting these goals in large-enrollment Astro 101 courses that typically rely on a lecture-only format (i.e. no lab component) may be difficult, especially given that students commonly have a significant 
lack of mathematical skills. These "lecture-only" courses often become primarily descriptive in nature, with the content covering a wide range of topics from motions of the sky, to planets and stars, to galaxies, expansion of the universe, and the Big Bang. The Mapping the Milky Way project offers a conceptually rich investigation that explicitly links many seemingly disparate concepts together to span a large portion of the course. Additionally, this investigation affords students a window into authentic data collection and analysis without overly burdening them with calculations.

In the Mapping the Milky Way project, each student uses a robotic radio telescope to collect spectral emission data from the cold neutral hydrogen gas (HI, pronounced "H one") that is found throughout the galaxy. Five lessons distributed throughout the course are used to help students meaningfully connect their observational data to basic astrophysics concepts, including the electromagnetic spectrum, atomic emission mechanisms, and Doppler shift. Students combine their data with others' in the class to analyze trends across the galaxy. Systematic analysis of Doppler shift trends, conducted in class, allows students to:

1. identify the direction of our orbit around the Milky Way's center,

2. discover that we live in a spiral galaxy, and

3. uncover that our galaxy's rotation curve is "flat," leading them to engage with the "missing mass" problem, for which they must invoke the concept of dark matter to resolve.

Through this rich scientific investigation, the Mapping the Milky Way project engages students in authentic data collection and analysis, allowing them to gain fascinating insights about our galaxy.

\section{Background}

The educational foundations of this project have their origins in the Green Bank Observatory's (GBO) "Radio Astronomer for a Day" program and the University of North Carolina (UNC) ERIRA (Educational Research in Radio Astronomy) program, both of which engage participants in HI data collection and analysis with an on-site manually-controlled forty-foot radio receiver. UNC introductory astronomy students gain experience with the Milky Way data analysis through the Dark Matter lab that uses archived ERIRA HI data. Additionally, there is an online Milky Way data simulator and mapping tool, as well as kinesthetic activities, from the European Hands-On Universe (EU-HOU) program. There are likely other educational Milky Way mapping efforts underway as well, but the purpose of this project is to adapt the project to the large-enrollment introductory college astronomy, "Astro 101," lecture format.

\section{Curriculum}

The Milky Way project adaptation for the large enrollment Astro 101 classroom described here has been piloted and refined for three semesters (Spring 2016, Fall 2016, and Spring 2017) at West Virginia University (WVU) by using the Green Bank 20-meter telescope operated remotely through the UNC Skynet Robotic Telescope Network. The data collection and analysis are distributed across five lectures over the course of the semester that link together as an authentic, unified, science investigation narrative. Here we elucidate the five mini-lessons to show how the project works at WVU and to provide other instructors with easily adoptable curriculum materials that can be inserted wherever appropriate for their own courses.

\section{Preperation Work: Getting Set Up With Skynet}

Instructors should request Skynet access at least a few weeks prior to the start of their semester. They will work with the UNC Skynet team to enter into a contract for telescope time, which is relatively inexpensive on its own, or free if purchased in conjunction with the UNC Skynet WebAssign online course. Once the semester begins, students receive personal accounts on Skynet and practice logging in and browsing the site. In-class instruction introduces students to the history and purpose of Skynet, as well as the online web interface. A scavenger hunt homework assignment 
guides students in an investigation of the relevant observation submission parameters on their own.

\section{Lesson 1: \\ Understanding Radio Waves, Atomic Emission, Frequency, and Collecting Data}

Interactive lectures introduce students to the concepts of telescopes, the electromagnetic spectrum (frequency, wavelength), and emission/absorption characteristics of atoms. While Astro 101 atomic emission mechanism lessons typically focus on energy level transitions of electrons, course material must also include the spin-flip transition by which the HI emission is produced so that students understand the radio frequency data they will be collecting and analyzing. There is no need to go into depth on the quantum mechanical property of "spin," but it can easily be explained with hand motions - thumbs up for aligned, a higher energy state, then one thumb flips to show the anti-aligned, lower energy state. The loss of energy goes into the creation of a radio photon with a wavelength of 21 centimeters, or 1420.41 MHz frequency. To help make the HI frequency more relatable, a lecture slide encourages students to make comparisons to the frequencies of radio stations available in cities, which occur in the 88-107 MHz range. The Green Bank 20-meter telescope acts like the radio in a car - except the telescope is "tuning in" to the 1420.41 MHz signal of the Milky Way.

For homework, students use Skynet to collect a 60 -second spectral scan along one direction of the Milky Way (see Figure 1). Each student is assigned a unique galactic longitude ${ }^{1}$ and a galactic latitude of zero degrees (i.e. they should look into the plane of the Milky Way). A 5-10-minute demonstration of the data analysis process is given during class. The instructor shows how to manipulate the spectrum graph to measure the frequency of the peaks to an accuracy of two decimal places. The

\footnotetext{
${ }^{1}$ Only galactic longitudes between about $0-230^{\circ}$ can be scanned by the Green Bank 20-meter, as the remaining portion of the galaxy is only visible from lower latitudes. This limitation can be leveraged in the service of learning to discuss the need for multiple telescopes located across the globe.
}

instructor also warns students that their data may have one or several peaks (for example, the data in Figure 1 shows three peaks), and that they should not ignore any smaller peaks that they detect. Students are told that if some peaks appear blended together, they must use their best judgment about how to analyze their data. This is a teachable moment to reinforce the idea that analyzing data is not always straightforward, and that sometimes scientists may draw different conclusions from the same data. Finally, the instructor motivates future analysis by saying that the class will use this data later in the semester to investigate some exciting topics. An online tutorial video is made available to remind students of the data analysis and submission process first shown in class.

Depending on the Skynet queue system and maintenance schedule of the Green Bank 20-meter telescope, the data may be taken immediately or within a week or so. ${ }^{2}$ Once the data is returned, students submit their results into an online Google form that automatically collates the entire class data into an easily-downloadable spreadsheet.

\section{Lesson 2: Doppler Shift}

To motivate the topic of Doppler Shift, students are asked: "If neutral hydrogen atoms are creating a very specific frequency of $1420.41 \mathrm{MHz}$, then why are we detecting a slight variation of frequencies?" Student ideas generally include: maybe we are not actually detecting hydrogen, or maybe there was an error in the instrument. After an interactive lecture on Doppler shift, a handout of the entire class data set is distributed to students, with the data organized according to galactic longitude. Students spend about five minutes in small groups differentiating redshifts and blueshifts. Students should identify that, for the most part, they observe blueshifts for galactic longitudes of $\sim 45-100^{\circ}$

\footnotetext{
${ }^{2}$ If the telescope is idle and the observation can occur immediately, one can watch the telescope move in-real time through the Skynet interface, offering an exciting demonstration that can be done during class.
} 
and redshifts for $\sim 160-245^{\circ 3}$ (Figure 2). At this point, students have not received instruction on what Galactic Coordinates are or what this newly discovered pattern means.

\section{Lesson 3: Mapping the Milky Way}

During the galaxies and cosmology unit, an interactive lecture on the properties of the Milky Way motivates upcoming data analysis by asking, "If you could never leave your house, how would you know what it looked like from the outside?" We discuss early attempts at mapping the Milky Way with star counting, then look at multi-wavelength images of the Milky Way to show that radio astronomy offers a useful way to peer through the interstellar gas and dust. Students refer to their collective radio data again, and recall that Doppler shift gives information about motion. The instructor leads students in a discussion of what could be moving, so students determine that the observed Doppler Shift pattern gives information about our own motion through the Milky Way. The "Milky Way Galaxy: Galactic Doppler Shift" quiz question from the University of Nebraska online astronomy suite helps to reinforce the conceptual reasoning. Finally, a think-pair-share question allows students to vote, based on findings from their own data, on which direction we must be orbiting around the galactic center - clockwise or counterclockwise as viewed from the North Galactic Pole. Students generally agree that their data implies clockwise, which is also consistent with findings from professional astronomers. This is a teachable moment to reinforce the nature of science: "did we get the correct answer, and do we trust our results?"

An addition to this lesson is an instructor-led investigation for evidence of spiral arms, starting with the question: "Do you observe any other trends in your data? Look closely at the frequencies you detect." The instructor shows a

\footnotetext{
${ }^{3}$ This finding is robust despite several potential sources of error. Namely, we have not corrected for the rotation of the Earth on its axis or its revolution around the Sun.
}

projection of the class data set to point out galactic longitude ranges that appear to have consistent frequencies. Some data sets are more obvious than others, but students are able to uncover that ranges in angular width of $10-20^{\circ}$ of galactic longitude generally have consistent frequencies. This represents an opportunity for doing some modeling and engaging students in critical thinking. Students are asked to consider if we are detecting a lot of little hydrogen gas clumps, or if we are detecting big, extended structures of hydrogen. Students deduce that, because the frequencies are consistent over larger regions of the sky, we must be identifying larger structures in the disk of the Milky Way. The instructor further facilitates critical thinking with the question: "What are some big, extended structures we see in other galaxies when observing in visible wavelengths?" Students then recognize that these large structures must be spiral arms! Through this guided investigation, a second, tentative conclusion students may be able to claim is that they have also discovered that our galaxy is a spiral galaxy. Again, this offers an important teachable discussion into the nature of science: "How confident are we in this claim?"

\section{Lesson 4: \\ Constructing a Rotation Curve}

By the end of the semester the course topics include dark matter, and again an investigation of students' data can motivate their learning. An important first step mimics the historical progression: constructing a rotation curve. To do this, calculations are needed of orbital speed and distance from the galactic center. These calculations involve simple trigonometry, and while they are included in the UNC Skynet labs, they may be too tedious for the lecture-setting of the large-enrollment, descriptive Astro 101 course. This lesson is a critical moment where instructors must choose how to balance the tension between Astro 101 students' limited math skills/phobias with the course goal of exposing them to the quantitative nature of science. Through the three semesters of piloting the Milky Way Mapping project at WVU, we have implemented a 


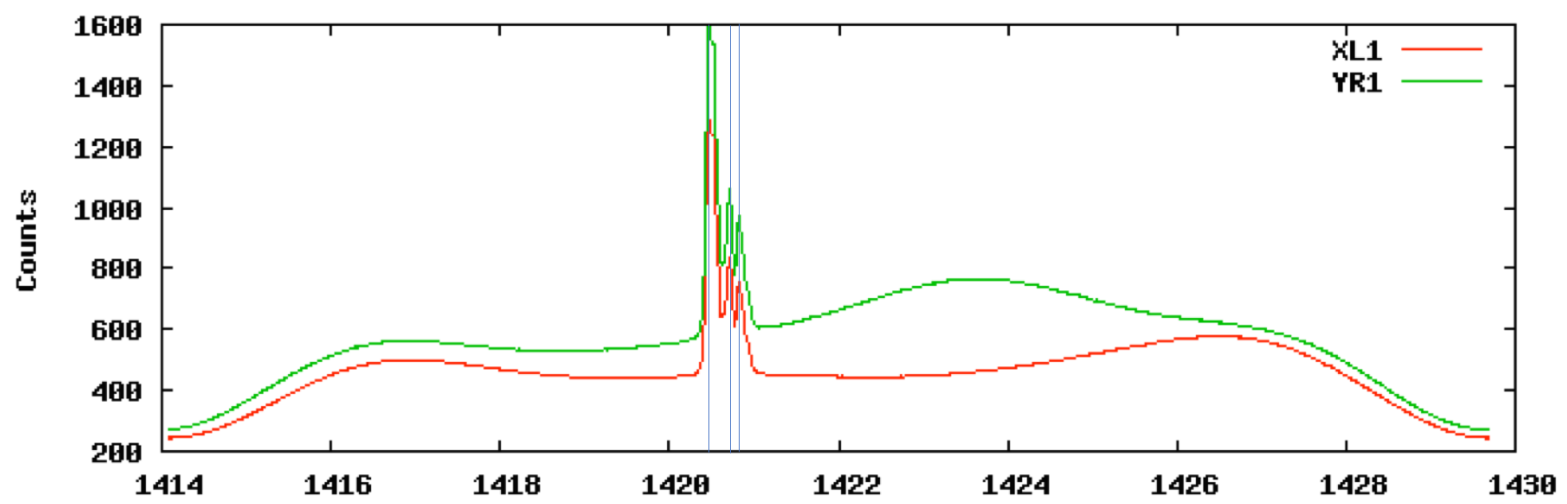

Figure 1. An Astro 101 student's HI data collected through Skynet with the Green Bank 20-meter radio telescope. The vertical axis represents a relative strength of detection, and the horizontal axis shows frequency in $\mathrm{MHz}$. The two curves arise from the two channels of the receiver. Vertical lines mark three detected peaks, potentially indicating three spiral arms of the Milky Way along this line of sight.

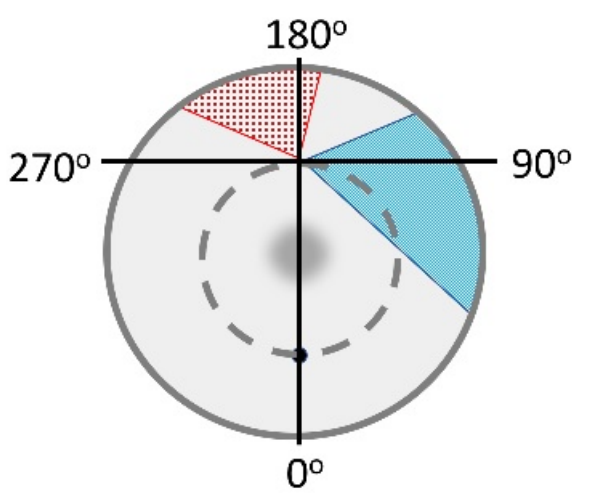

Figure 2. For the most part, students observe blueshifts between a galactic longitude of $\sim 45-100^{\circ}$ and redshifts between $\sim 160-245^{\circ}$. Blue shade represent blueshift, red checkers represent redshift.

compromise between these competing demands: the instructor performs the calculations ahead of time, then passes out a printout of the results to students during class and leads an interactive discussion designed to unpack the different mathematical steps undertaken to arrive at the results provided in the handout. In an effort to ensure that students remain intellectually engaged during this quantitative portion of the lesson, and provide formative feedback that students are understanding the basic purpose of the calculations, the instructor poses a series of think-pair-share questions throughout this interactive lecture (Mazur, 1997).
The key to calculating speeds and distances from data at different galactic longitudes is to use the frequency of the most-redshifted peak along each line of sight (for example, the peak farthest to the left in Figure 1). Assuming that the most-redshifted peak corresponds to hydrogen gas that is moving with only a radial velocity (with no transverse velocity), the observed velocity is the true relative velocity between the hydrogen gas and our solar system. Correcting for our own solar system's velocity, the component of our solar system's motion along that line of sight is added to the observed velocity. Additionally, the hydrogen cloud that creates the most-redshifted peak must be at a particular orbital radius, as shown in the lecture slides in Figure 3. Since we know the galactic longitude of the observation and the distance from our solar system to the galactic center, simple trigonometry can be used to determie this hydrogen cloud's orbital radius. A full conceptual and mathematical derivation of these concepts can be found in the text Galactic and Extragalactic Radio Astronomy (Kellermann and Verschuur, 1988). Again, all of these steps are unpacked and reinforced by the instructor in class with images, graphs, analogies, and think-pair-share questions (all available upon request to the first author).

Plotting the results of these simple calculations on a rotational velocity curve provides a graph (Figure 4) that is comparable to published data (for 


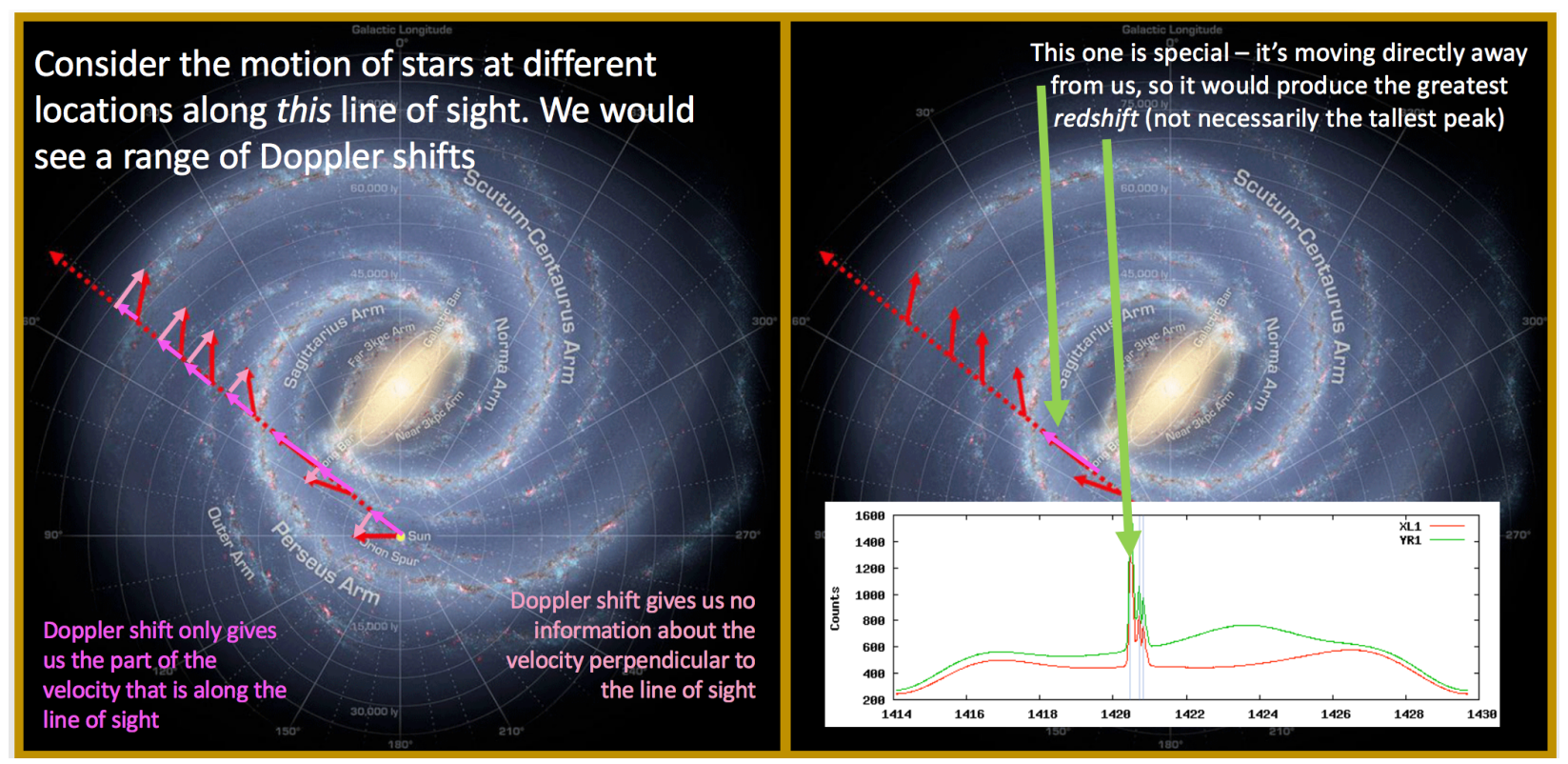

Figure 3. Two lecture slides that show why the most-redshifted signal is special. We assume that the most-redshifted signal comes from a hydrogen cloud that is moving tangent to a very particular orbital radius. Since we know the galactic longitude of the observation and the distance from our solar system to the galactic center, simple trigonometry can be used to determine this hydrogen cloud's orbital radius.

example, Clemens 1985). This method of using the most red-shifted peak only works for galactic longitudes up to $90^{\circ}$, and measurements at small longitudes show some confusion amidst material at the galactic center, i.e. Figure 4 shows dips at roughly 5,000 and 8,000 lightyears, radii that are within the central galactic bulge where motions of material may deviate from overall galactic trends. Despite these limitations, the rotation curve created with the class data shows the rotational velocity of hydrogen gas far from the galactic center trends towards a constant value of approximately 200 $\mathrm{km} / \mathrm{s}$, very close to the accepted value of $220 \mathrm{~km} / \mathrm{s}$. Instructor-led class discussions compare the results from students' data to Keplerian motion concepts that were covered during previous lessons on gravity and solar system dynamics. From their own data, students find that the Milky Way's rotation curve is relatively flat with distance, so mass must be more spread out than in our solar system.

\section{Lesson 5:}

\section{Evidence for Dark Matter}

To investigate this new insight into the mass distribution of our galaxy, students' data can further be used to calculate the mass enclosed within each orbital radius. Building on students' prior learning of gravity, Equation 1 is introduced in lecture. This equation is used ahead of time by the instructor to calculate the mass enclosed for each galactic longitude; however, since previous calculations only apply to longitudes less than 90 degrees, the mass enclosed can only be calculated out to the orbital radius of the Solar System. Therefore, the instructor adds archived measurements of objects farther from the Milky Way's center, such as the Large and Small Magellanic Clouds (consistent with the UNC Skynet Lab). Figure 5 shows results from the Spring 2017 semester, which is also used as a lecture slide. While there is still a large amount of information missing about the outer galaxy, the conceptual lesson is clear: there are several hundred billion solar masses of material that are unseen. This so-called "missing mass problem" leads students to determine that there must be a vast amount of unseen mass, i.e. dark matter, surrounding the Milky Way.

$$
M=\frac{V^{2} \cdot R}{G}
$$




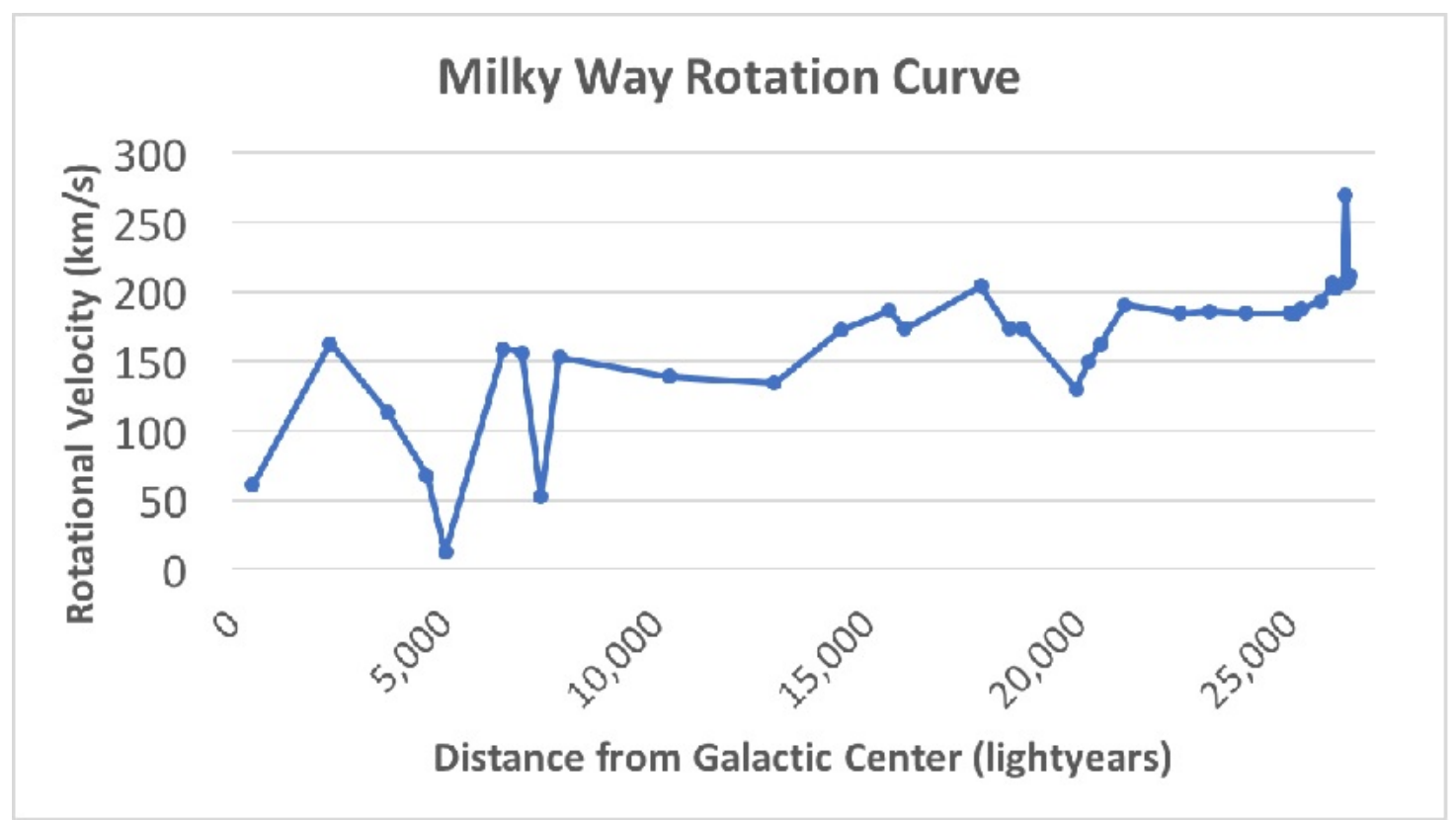

Figure 4. The Milky Way rotation curve constructed with students' data is comparable to professional published data. It shows a trend toward a constant rotation rate approximately $200 \mathrm{~km} / \mathrm{s}$, very close to the accepted value of $220 \mathrm{~km} / \mathrm{s}$.

Approximately half of lecture time is used for Lesson 5, and the remaining class period is used for students to work in groups on the "Dark Matter" Lecture-Tutorial (Prather et al., 2012). The tutorial again leads students through the same reasoning processes they were able to do with their own data, thereby reinforcing the concepts of rotation curves, enclosed mass, and dark matter.

\section{Final Thoughts}

To explicitly support increased student interest and incorporate confidence-building into the course, throughout the lessons described above, the instructor pauses to encourage students to reflect that they collected this data. By the end of the dark matter lesson, the instructor recaps the entire conceptual arc of the project to remind students of how hard they worked to get to this point (i.e. they had to learn about radio waves, how to use a telescope, how to analyze data, understand Doppler shift, look for patterns, use critical reasoning, etc.). The instructor explicitly reminds students of the breadth of conclusions their data have allowed them to draw. With a collection of simple 60 -second radio scans, students were able to determine our direction of rotation around the center of the Milky Way, determine that we live in a spiral galaxy, and provide evidence in support of the claim that our galaxy is filled and surrounded by dark matter. These are profound discoveries and claims about our galaxy that can be made by novice students.

Basic assessment of students' perceptions of this experience was captured with three five-point agree/disagree Likert scale items at the end of the semester. For $\mathrm{N}=246$ students, $73 \%$ agreed that conducting this research enhanced their interest and enjoyment of the course, 57\% agreed that this experience enhanced their confidence in doing scientific research, and $62 \%$ agreed that they had greater understanding of course content. A typical student quote from an open-ended option to explain their response choices is:

"Using the 20-meter telescope showed me how to relate my findings that I got to real data others have gotten. Using the telescope and being able to gather my own data and see that everyone else was using it correctly, boosted my confidence, and made me realize how doing 


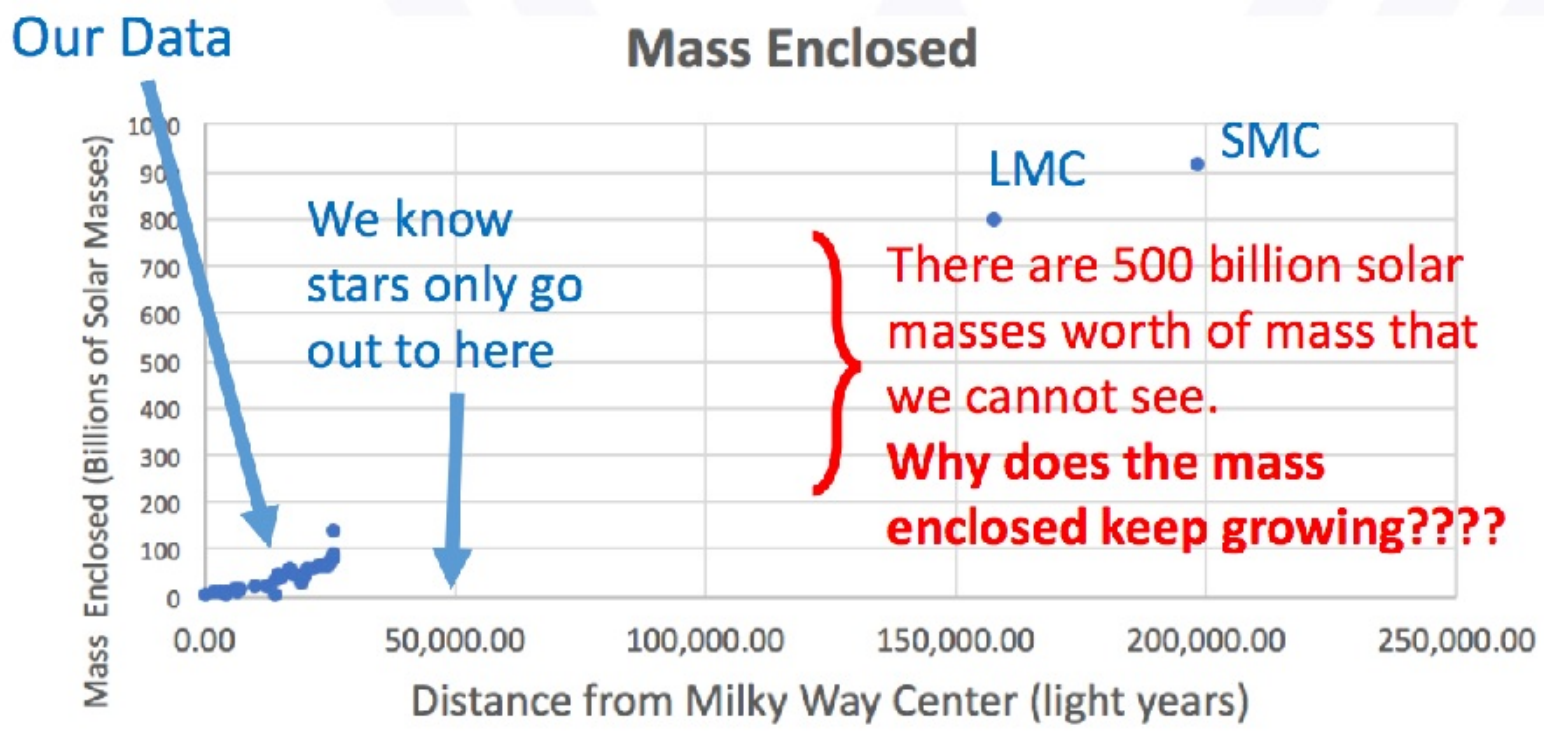

Figure 5. Lecture slide combining class data with LMC and SMC data to construct an enclosed mass curve for the Milky Way. The rise in enclosed mass beyond 50,000 lightyears implies the existence of dark matter.

scientific research could be so helpful." (WVU Astro 101 Student)

While the assessment was self-report and post-reflective, it implies that the Mapping the Milky Way project can help instructors advance many of the recommended Astro 101 goals. In the future, we plan to implement more-robust assessment with pre- and post-instruction conceptual questions, as well as a controlled comparison experiment in which one section engages in the data-motivated Mapping the Milky Way project and one section is only told about the concepts. We invite others to test this curriculum with their Astro 101 courses and help to more thoroughly evaluate its impact on students. Copies of lecture slides, tutorial videos, assessment questions, and calculations are all available by contacting the first author. Data and analysis templates are also available online at the UNC Skynet Lab website.

\section{Acknowledgements}

The authors would like to thank the Green Bank Observatory for providing access to the 20-meter telescope through Skynet and the WVU Astro 101 students who participated in providing feedback on this project.

\section{References}

Clemens, D. P. (1985). Massachusetts-Stony Brook Galactic plane CO survey-The Galactic disk rotation curve. The Astrophysical Journal, 295:422428.

Kellermann, K. and Verschuur, G. (1988). Galactic and extragalactic radio astronomy.

Mazur, E. (1997). Peer Instruction: A user's manual.

Partridge, B. and Greenstein, G. (2003). Goals for "Astro 101": Report on workshops for department leaders. Astronomy Education Review, 2(2):46-89.

Prather, E. E., Brissenden, G., Adams, J. P., and Slater, T. F. (2012). Lecture-tutorials for introductory astronomy. Addison-Wesley, 3 edition.

Rudolph, A. L., Prather, E. E., Brissenden, G., Consiglio, D., and Gonzaga, V. (2010). A national study assessing the teaching and learning of introductory astronomy part II: The connection between student demographics and learning. Astronomy Education Review, 9(1). 